\title{
Fabrication of Upended Taper-Shaped Cuprous Thiocyanate Arrays on a Copper Surface at Room Temperature
}

\author{
Jiasheng Xu and Dongfeng Xue* \\ State Key Laboratory of Fine Chemicals, Department of Materials Science and Chemical \\ Engineering, School of Chemical Engineering, Dalian University of Technology, 158 Zhongshan \\ Road, Dalian 116012, P. R. China \\ *Corresponding author. E-mail: dfxue@chem.dlut.edu.cn
}

Supporting Information 


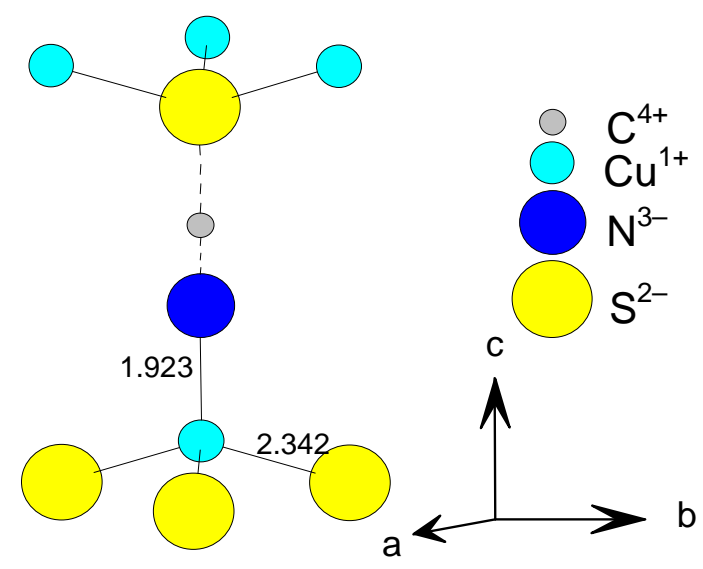

Figure S1. Part of $\beta$-CuCNS crystal structure, which clearly shows the different types bonds in the crystal structure. Dashed lines represent type (I) bonds that have been formed before the crystallization; solid lines represent type (II) bonds that formed during the crystallization. The two dates are in the unit of angstrom $(\AA)$.

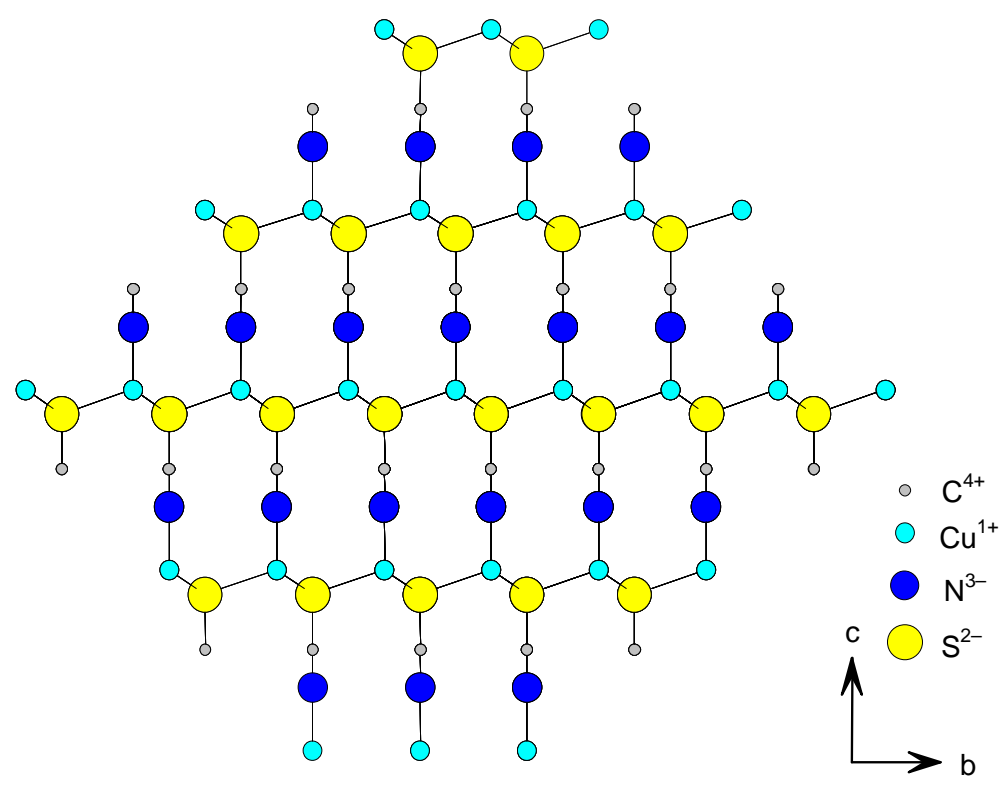

Figure S2. Projection of $\beta$-CuCNS structure along $a$-axis, i.e. the $\{100\}$ planes lie in the plane of the paper. 


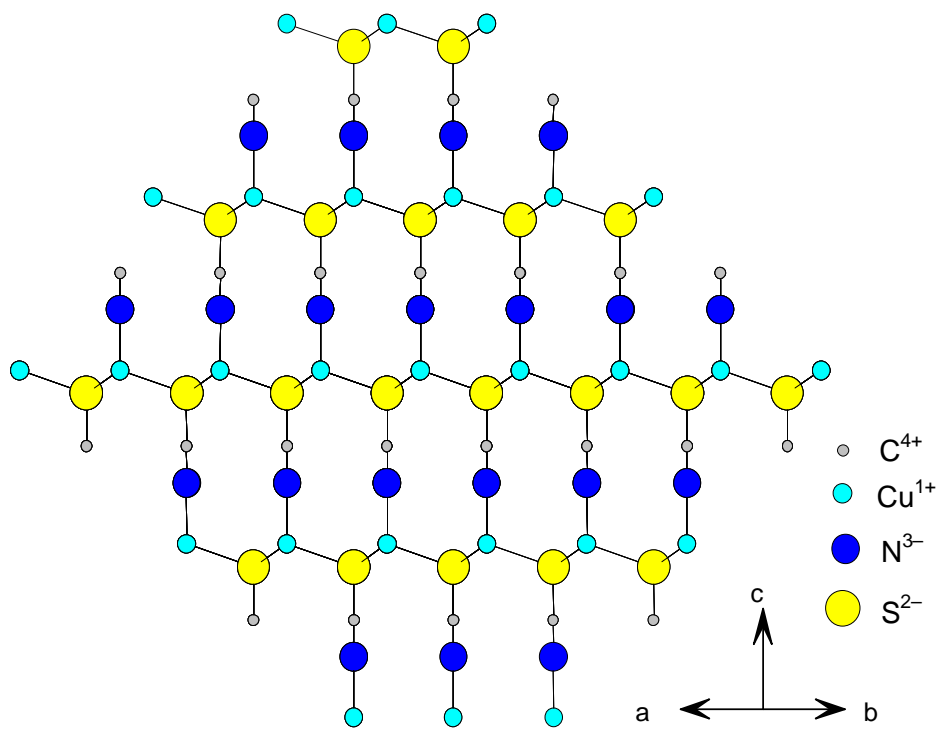

Figure S3. Projection of $\beta$-CuCNS structure along [110] direction, i.e. the $\{110\}$ planes lie in the plane of the paper.

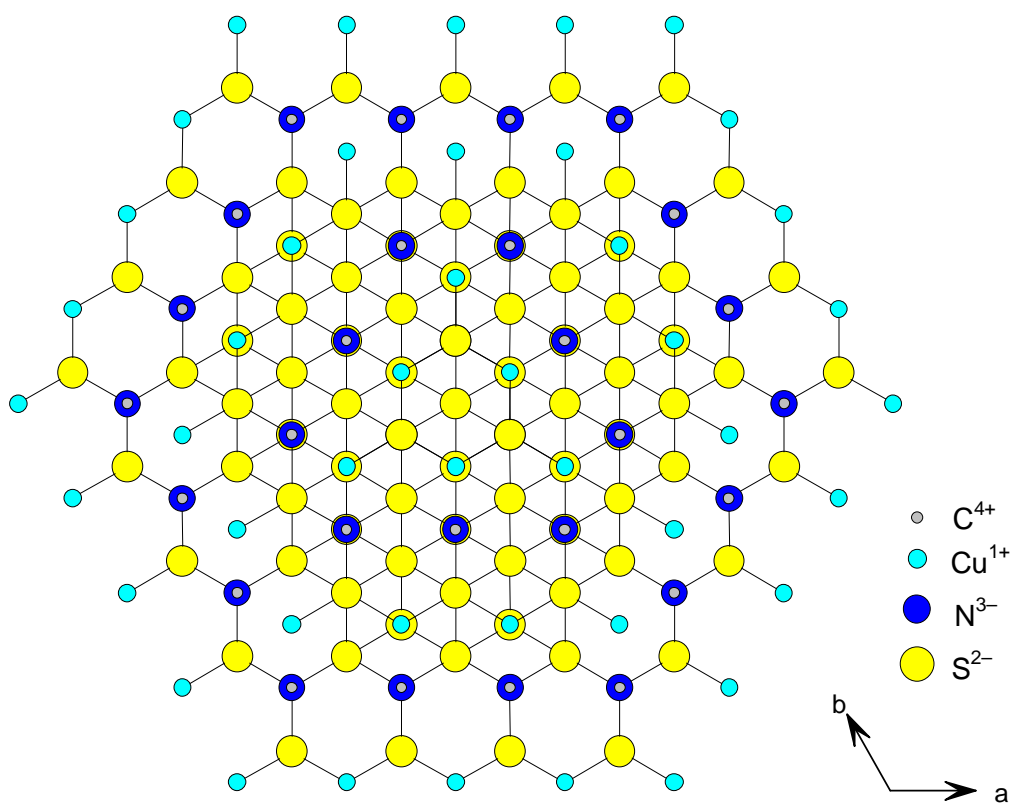

Figure S4. Projection of $\beta$-CuCNS structure along $c$-axis, i.e. the $\{001\}$ planes lie in the plane of the paper. 

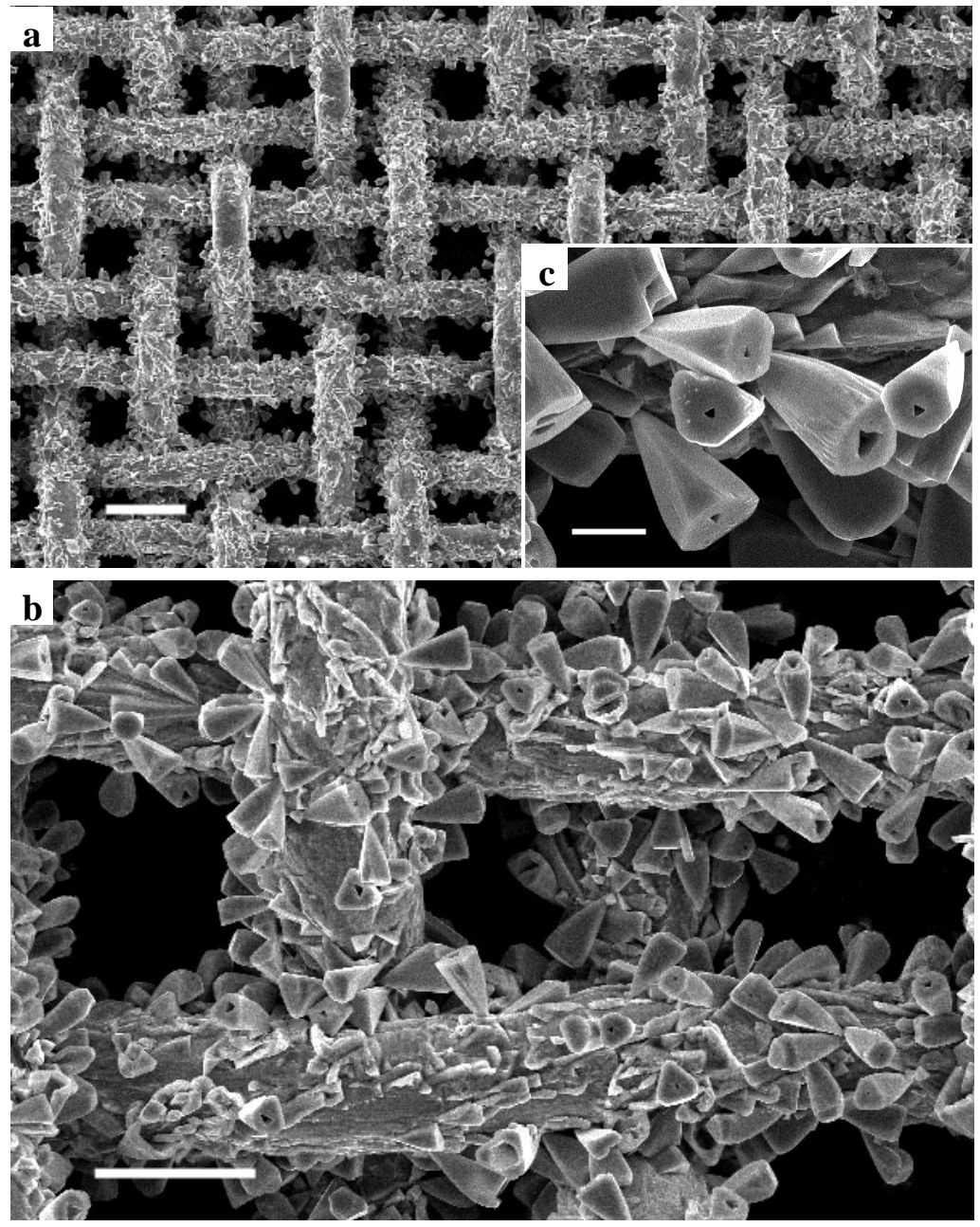

Figure S5. SEM images of cuprous thiocyanate crystals on a copper mesh substrate (experimental conditions: $1.0 \mathrm{~mL} \mathrm{NH}_{4} \mathrm{CNS}(0.2 \mathrm{M})+1.0 \mathrm{~mL}$ formamide $+8.0 \mathrm{~mL} \mathrm{H}_{2} \mathrm{O}$ at room temperature for 12 days). (a) Panoramic morphologies, scale bar $=100 \mu \mathrm{m}$. (b and c) Detailed view with high magnifications, which shows an inside hole on the top of upended taper-shaped cuprous thiocyanate crystals. Scale bar $=50$ and $10 \mu \mathrm{m}$, respectively. 

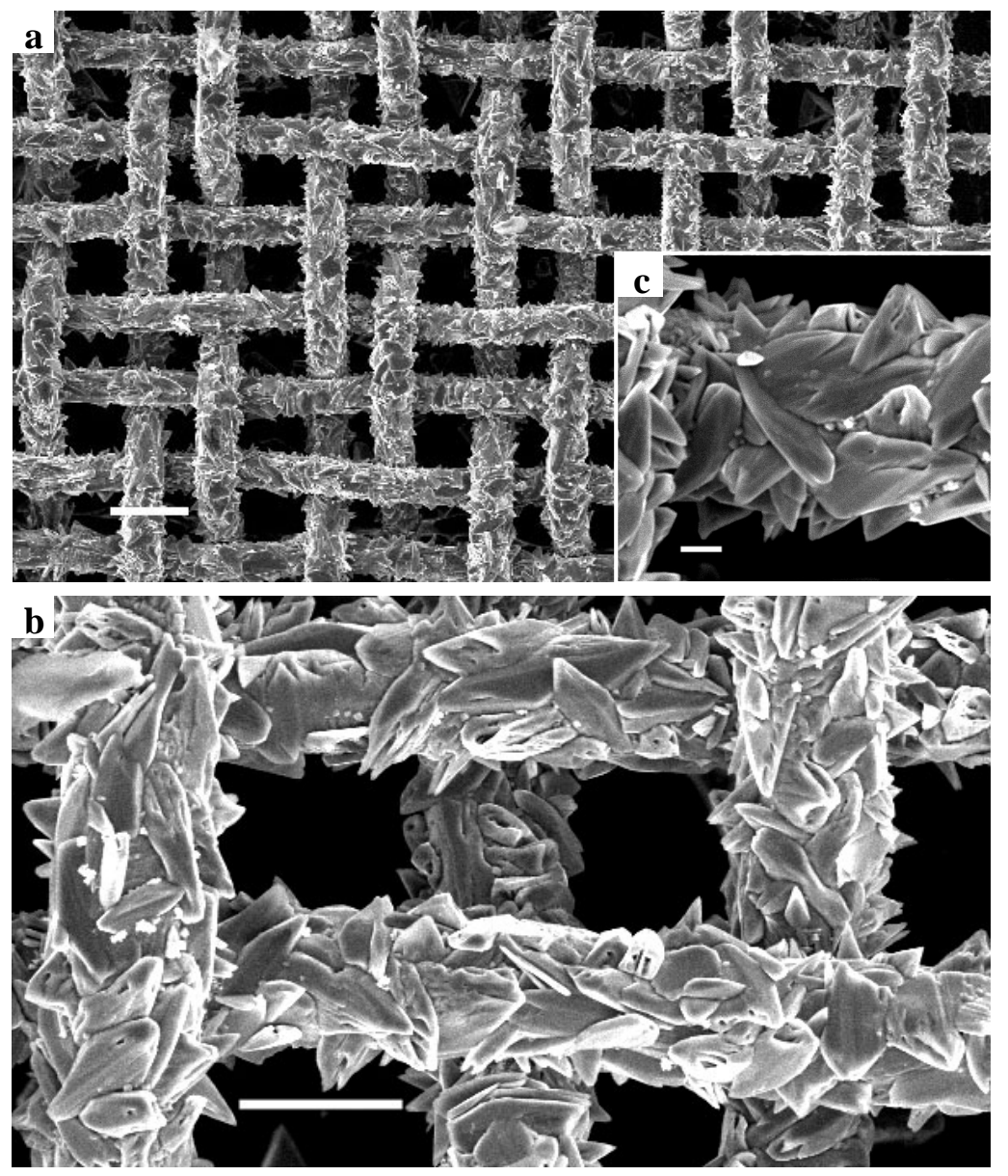

Figure S6. SEM images of cuprous thiocyanate crystals on a copper mesh substrate (experimental conditions: $1.0 \mathrm{~mL} \mathrm{NH}_{4} \mathrm{CNS}(0.2 \mathrm{M})+4.0 \mathrm{~mL}$ sodium dodecylsulfate $(10.0 \mathrm{mM})$ at room temperature for 12 days). (a) Panoramic morphologies, scale bar $=100 \mu \mathrm{m}$. (b and c) Detailed view with high magnifications. Scale bar $=50$ and $10 \mu \mathrm{m}$, respectively. 

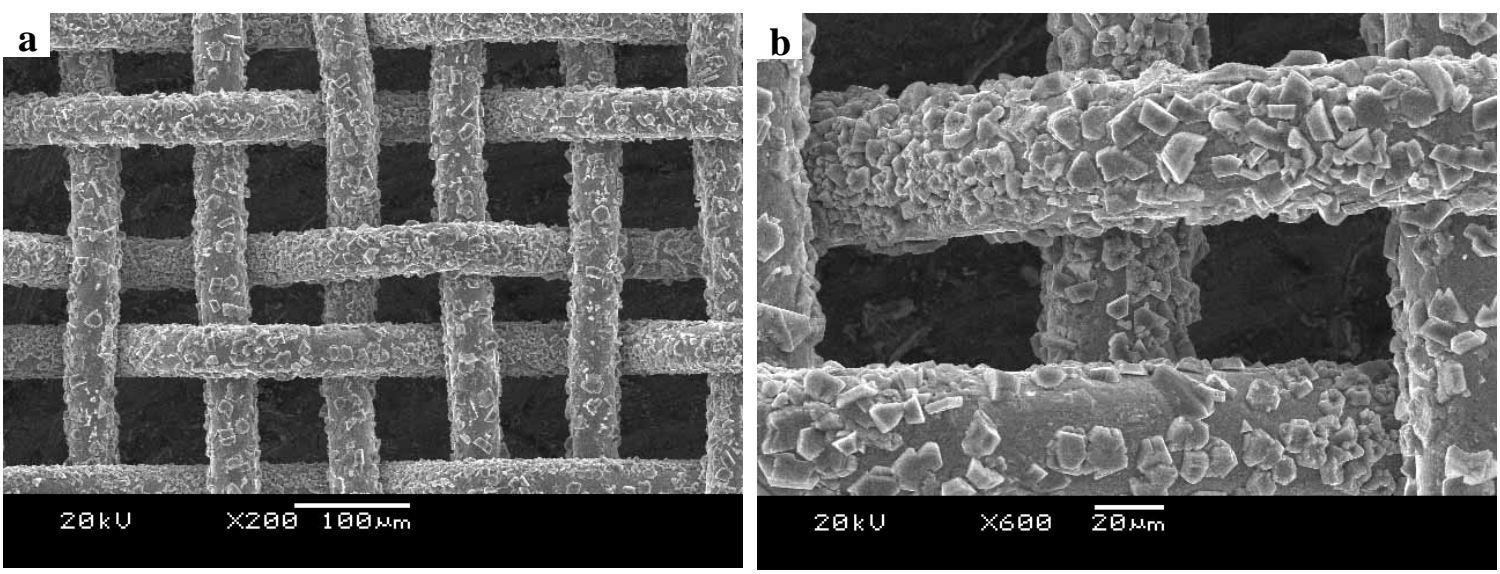

Figure S7. SEM image of $\beta$-CuCNS particles on the copper substrate with irregular morphology in the absence of formamide or ammonia (experimental conditions: $1.0 \mathrm{~mL} \mathrm{NH}_{4} \mathrm{CNS}(0.2 \mathrm{M})+$ $4.0 \mathrm{~mL} \mathrm{H}_{2} \mathrm{O}$ at room temperature for 12 days): (a) a low magnification view and (b) a high magnification view.

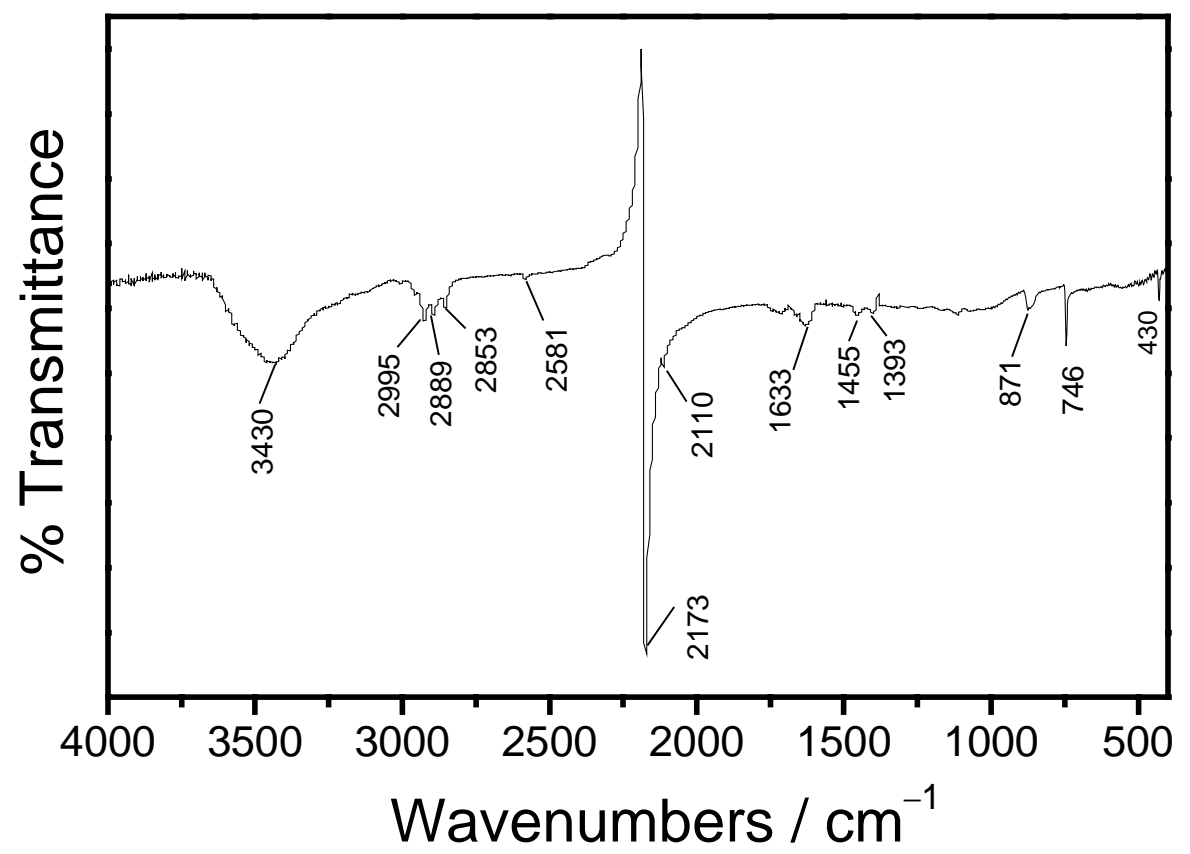

Figure S8. FT-IR spectra of the as-prepared $\beta$-CuCNS samples, the band at $2173 \mathrm{~cm}^{-1}$ is assigned to the $\mathrm{CN}$ stretching modes in the $\beta$-CuCNS crystal lattice, which coincides well with that reported in the literature. ${ }^{18}$ 